\title{
Highly Sensitive Fluorescence Detection of Avidin/Streptavidin with an Optical Interference Mirror Slide
}

\author{
Mitsuru YASUdA and Takuo AкImoto ${ }^{\dagger}$ \\ School of Bioscience and Biotechnology, Tokyo University of Technology, 1404-1 Katakura, Hachioji, \\ Tokyo 192-0982, Japan
}

\begin{abstract}
This paper presents highly sensitive fluorescence detections of avidin and streptavidin using an optical interference mirror (OIM) slide consisting of a plane mirror covered with an optical interference layer. Compared with a common glass slide, the OIM slide can enhance the fluorescence from a dye by more than 100-fold. We fabricated an OIM slide by depositing an optical interference layer of $\mathrm{Al}_{2} \mathrm{O}_{3}$ on an $\mathrm{Ag}$ mirror. To enhance the fluorescence maximally, the optimal thickness of the $\mathrm{Al}_{2} \mathrm{O}_{3}$ layer was estimated from optical interference theory. For detections of protein, avidin/streptavidin labeled with fluorescein, $\mathrm{Cy} 3$, and $\mathrm{Cy} 5$ were detected with biotin immobilized on an OIM slide with the optimal $\mathrm{Al}_{2} \mathrm{O}_{3}$ thickness. We achieved a sensitivity improvement of more than 50-fold, comparing with a glass slide. Such a high degree of improvement would be a significant contribution to further progress in biomedical research and medical diagnostics.
\end{abstract}

(Received June 4, 2012; Accepted September 12, 2012; Published October 10, 2012)

\section{Introduction}

Fluorescent labeling has dramatically increased the efficiency in the detection of target molecules. In particular, microarray technology allows parallel analysis of targets through specific molecule recognition of probes arrayed in a high-density configuration on a solid substrate. Typical DNA microarrays, ${ }^{1}$ and protein arrays, ${ }^{2}$ enable clinical diagnostics ranging from the discovery of disease-related genes to the detection of biomarkers. The sensitivity of target detection based on such fluorescent labeling is limited owing to the inherent fluorescence signals of a dye. Because an enhancement of fluorescence leads to improved sensitivity, enhancing fluorescence is becoming increasingly important for advances in contemporary genomic and proteomic research.

A number of substrates including three-dimensional micro/nanostructured surfaces have been developed to enhance fluorescence. ${ }^{3-8}$ Fluorescence enhancement based on the localized surface plasmon resonance of a silver island structure is well known as a typical fluorescence enhancement technique. Such a structure can improve the sensitivity of target detection up to approximately 50-fold compared with that of a flat control surface. $^{9-11}$ In addition, porous and pillar structures with large surface areas can improve the sensitivity up to approximately 7-fold, ${ }^{12-14}$ and 34-fold, ${ }^{15-17}$ respectively.

Among various substrates, we focused on an optical interference mirror (OIM) slide that consists of a plane mirror covered with an optical interference (OI) layer. The strategy using the OIM slide is based on double interference of both the excitation light and the fluorescence in the OI layer. Hall's group first reported that the fluorescence from rhodamine B on an OIM slide consisting of an Ag mirror and an OI layer of $\mathrm{LiF}$

† To whom correspondence should be addressed.

E-mail: akimoto@be.teu.ac.jp achieved an enhancement of more than 100-fold. ${ }^{18-21}$ Although it depended on the OI layer thickness, this enhancement exceeding two orders of magnitude has attracted much attention. The large magnitude of the enhancement is mainly explained by the waveguide mode in the OI layer and the surface plasmon induced on the mirror surface. ${ }^{18}$

In general, it is inadequate to only enhance the fluorescence as a substrate for biomolecular analysis. An ideal substrate should ensure facility, reproducibility, and productivity for the fabrication of a surface structure. Many reported substrates including the aforementioned three-dimensional micro/nanostructured surfaces involve some controls, such as the size, shape, and density of surface structures. Although the fabrication of such substrates is difficult, the OIM slide satisfies all of the requirements. The only significant control factor in the fabrication of the OIM slide is the thickness of the OI layer. Thus, the OIM slide is expected to be a promising alternative to traditional substrates for biomolecular analysis.

We previously succeeded in enhancing the fluorescence from rhodamine $\mathrm{B}$ up to 200-fold by using an OIM slide that consists of an $\mathrm{Ag}$ mirror and an OI layer of $\mathrm{Al}_{2} \mathrm{O}_{3} .^{22,23}$ This $\mathrm{Ag} / \mathrm{Al}_{2} \mathrm{O}_{3}$ structure has also been reported by another group. ${ }^{24,25}$ Other reported substrates based on OI include substrates with an $\mathrm{Si} / \mathrm{SiO}_{2}$ structure, ${ }^{26-28}$ and those with a dielectric multilayered structure. ${ }^{29-31}$ However, the enhancement with these substrates was less than 20-fold. Therefore, we predicted that the presence of an Ag mirror is essential for high-sensitivity detection of biomolecules on an OIM slide.

In this study, we demonstrated highly sensitive fluorescence detection of protein using an OIM slide fabricated with $\mathrm{Ag}$ and $\mathrm{Al}_{2} \mathrm{O}_{3}$ to improve the sensitivity of biomolecular analysis. Avidin and streptavidin labeled with fluorescein, $\mathrm{Cy} 3$, and $\mathrm{Cy} 5$ were detected with biotin immobilized on the OIM slide. The detection sensitivity of the avidin/streptavidin using the OIM slide was evaluated by a comparison with that obtained using a conventional glass slide. 


\section{Experimental}

\section{Reagents and proteins}

(3-Aminopropyl)trimethoxysilane (APTMS) and biotin were purchased from Sigma-Aldrich Co. (MO). A sulfo-NHS biotin derivative (biotin $N$-hydroxy-sulfosuccinimide ester) was purchased from Dojindo Laboratories (Kumamoto, Japan). In this paper, to avoid confusion, biotin and the sulfo-NHS biotin derivative are referred to as non-activated biotin and NHS-activated biotin, respectively. Fluorescein-labeled avidin was procured from Calbiochem (CA). Cy3- and Cy5-labeled streptavidin were obtained from GE Healthcare UK Ltd. (Buckinghamshire, England). Bovine serum albumin (BSA) and 2-amino-2-hydroxymethyl-1,3-propanediol (Tris) were obtained from Wako Pure Chemical Industries, Ltd. (Osaka, Japan). Tris- $\mathrm{HCl}$ buffer $(10 \mathrm{mM}, \mathrm{pH}$ 7.4) was prepared as a buffer for analysis.

\section{Fabrication of the OIM slide}

Microscope glass slides were first sonicated in ethanol for $1 \mathrm{~h}$, rinsed with $\mathrm{H}_{2} \mathrm{O}$, and then dried in an oven at $100^{\circ} \mathrm{C}$. The OIM slide was fabricated according to a procedure described previously. ${ }^{22,23}$ Briefly, the OIM slides were fabricated by depositing $\mathrm{Cr}$ as an adhesive, $\mathrm{Ag}$ as a mirror, and $\mathrm{Al}_{2} \mathrm{O}_{3}$ as an $\mathrm{OI}$ layer onto cleaned glass slides. The thicknesses of the $\mathrm{Cr}$ and Ag layers were $15 \mathrm{~nm}$ and $1 \mu \mathrm{m}$, respectively.

\section{Design of the $\mathrm{Al}_{2} \mathrm{O}_{3}$ thickness}

Because the thickness of the $\mathrm{Al}_{2} \mathrm{O}_{3}$ layer that provides the maximum fluorescence enhancement varies among dyes, the optimal $\mathrm{Al}_{2} \mathrm{O}_{3}$ thickness was determined for each dye. From our previous results, it was possible to estimate the optimal $\mathrm{Al}_{2} \mathrm{O}_{3}$ thickness on the basis of OI theory: $n_{2} d \cos \theta_{2}=(2 m+1) \lambda / 4$, $(m=0,1,2 \ldots) .^{23}$

The refractive indices, $n_{1}$ of air and $n_{2}$ of $\mathrm{Al}_{2} \mathrm{O}_{3}$, were assumed to be 1 and $1.62,{ }^{32}$ respectively. When the incident angle, $\theta_{1}$, was assumed to be $0^{\circ}$, the refraction angle, $\theta_{2}$, was calculated from Snell's law. $\lambda$ is the emission wavelength of a dye and $d$ the $\mathrm{Al}_{2} \mathrm{O}_{3}$ thickness. In this study, $m=0$ was adopted corresponding to the first bright fringe.

The optimal $\mathrm{Al}_{2} \mathrm{O}_{3}$ thicknesses for fluorescein (Excitation (Ex), 490 nm; Emission (Em), 514 nm), Cy3 (Ex, 550 nm; Em, $570 \mathrm{~nm}$ ), and Cy5 (Ex, $649 \mathrm{~nm}$; Em, $670 \mathrm{~nm}$ ) were determined to be 79,88 , and $103 \mathrm{~nm}$, respectively. Thus, the $\mathrm{Al}_{2} \mathrm{O}_{3}$ thickness of $80 \mathrm{~nm}$ for the detection of fluorescein-avidin, $90 \mathrm{~nm}$ for Cy3-streptavidin, and $100 \mathrm{~nm}$ for Cy5-streptavidin were used.

\section{Immobilization of biotin}

The OIM slides were aminated by incubating in 1\% APTMS diluted with $\mathrm{H}_{2} \mathrm{O}$ for $1.5 \mathrm{~h}$, washing in $\mathrm{H}_{2} \mathrm{O}$ for $10 \mathrm{~min}$, and then drying in an oven for $10 \mathrm{~min}$ at $110^{\circ} \mathrm{C}$. NHS-activated biotin dissolved with $\mathrm{H}_{2} \mathrm{O}$ was arrayed onto the aminated OIM slides using a spotter (BioChip Arrayer, PerkinElmer). The spotting conditions were a distance of $1 \mathrm{~mm}$ between the centers of adjacent spots and a solution volume of $10 \mathrm{~nL}$. The OIM slides were immersed in $10 \mathrm{mg} / \mathrm{mL}$ of BSA dissolved in $\mathrm{H}_{2} \mathrm{O}$, which acts as a blocking reagent. After $30 \mathrm{~min}$, the OIM slides were washed in $\mathrm{H}_{2} \mathrm{O}$ for $30 \mathrm{~min}$ and then dried at room temperature.

To verify the immobilization of biotin, the same process was performed with non-activated biotin dissolved with $\mathrm{H}_{2} \mathrm{O}$.

\section{Detection of avidin/streptavidin}

Two methods were employed to evaluate the sensitivity of the avidin/streptavidin detections with the OIM slide. In this study,

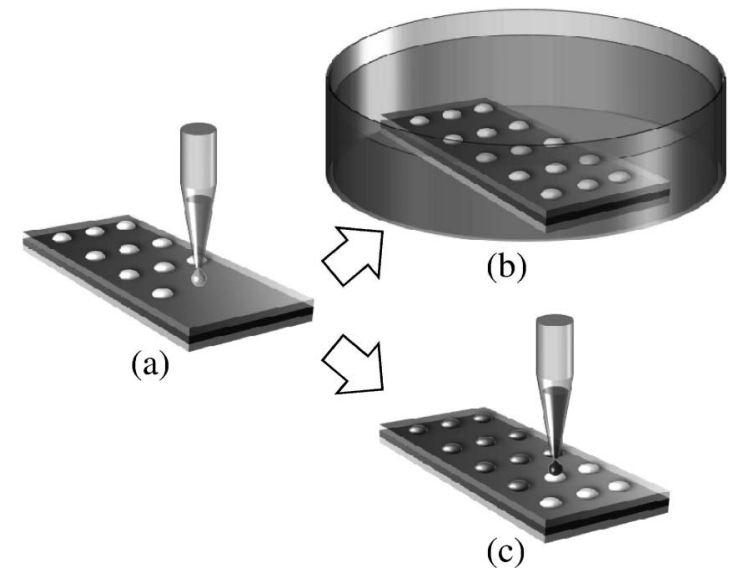

Fig. 1 Protocols of avidin/streptavidin detections. (a) Spotting of NHS-activated biotin on the OIM slide. (b) Immersion into a dyelabeled avidin/streptavidin solution. Solution assay. (c) Spotting of a dye-labeled avidin/streptavidin solution. Spotting assay.

these methods are referred to as a solution assay and a spotting assay. The solution assay was used to examine both the concentration dependence of the immobilized biotin and the detection sensitivity. Similarly, the spotting assay was used to examine the concentration dependence of the dye-labeled avidin/streptavidin and the detection sensitivity. Detailed protocols for each assay method are described below and their schemes are shown in Fig. 1.

\section{Solution assay}

The solution assay is a common analytical method for biomolecules. NHS-activated biotin was first arrayed onto the aminated OIM slides at concentrations of $0-10 \mu \mathrm{g} / \mathrm{mL}$. The biotin-immobilized OIM slides were then immersed in 1.0 or $10 \mu \mathrm{g} / \mathrm{mL}$ of the dye-labeled avidin/streptavidin solution dissolved in a Tris- $\mathrm{HCl}$ buffer. After $30 \mathrm{~min}$, the OIM slides were washed in the Tris- $\mathrm{HCl}$ buffer for $30 \mathrm{~min}$, rinsed with $\mathrm{H}_{2} \mathrm{O}$, and then dried at room temperature.

\section{Spotting assay}

The spotting assay is characterized by examining a single substrate concerning the concentration dependence of targets. Because this method does not require the preparation of several substrates for each target concentration, a more detailed quantitative estimation of the assay compared with that using common analytical methods, such as the solution assay, is possible.

After $10 \mu \mathrm{g} / \mathrm{mL}$ of NHS-activated biotin was first arrayed onto aminated OIM slides, $10 \mathrm{~nL}$ of a dye-labeled avidin/streptavidin solution was then directly spotted onto the biotin spot area of the biotin-immobilized OIM slides at concentrations of $0-1.0$ or $0-10 \mu \mathrm{g} / \mathrm{mL}$ in the Tris- $\mathrm{HCl}$ buffer. To bind dye-labeled avidin/streptavidin to the immobilized biotin, the OIM slides were immersed in the Tris- $\mathrm{HCl}$ buffer. After $30 \mathrm{~min}$, the OIM slides were washed in the Tris- $\mathrm{HCl}$ buffer for $30 \mathrm{~min}$, rinsed with $\mathrm{H}_{2} \mathrm{O}$, and then dried at room temperature.

To demonstrate that the fluorescence from the dye was not due to nonspecific adsorption of dye-labeled avidin/streptavidin, the same process was performed with the non-activated biotin-arrayed OIM slides. 


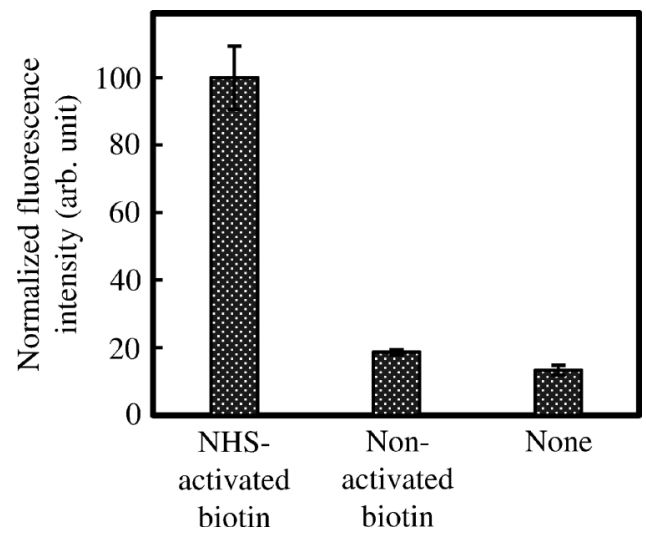

Fig. 2 Immobilization of biotin onto the OIM slide surface. The fluorescence intensity with the NHS-activated biotin-arrayed surface was normalized as 100 . The fluorescence intensity was averaged for five spots. Error bars indicate the standard deviation.

\section{Fluorescence imaging}

The fluorescence from the dye was detected with a fluorescence scanner (Typhoon 9410, GE Healthcare UK Ltd.). The dye was excited with a laser, and the fluorescence was detected using a bandpass filter; a 488-nm laser and a 520-nm filter for fluorescein; a 532-nm laser and a 580-nm filter for Cy3; a 633-nm laser and a 670-nm filter for Cy5. The obtained fluorescence images were analyzed with software (ImageQuant TL) provided by the manufacturer of the fluorescence scanner. The sensitivity of the avidin/streptavidin detections using the OIM slide was evaluated by a comparison with that using a glass slide.

\section{Results and Discussion}

\section{Verification of biotin immobilization}

First, the immobilization of biotin onto the OIM slide was verified. The immobilization was demonstrated by comparing the fluorescence intensity obtained for three different surfaces: the NHS-activated biotin-arrayed surface, the non-activated biotin-arrayed surface, and an unarrayed surface. For the NHS-activated biotin-arrayed surface, it was expected that the NHS-activated biotin would bind to the amino group on the OIM slide surface via amide binding. On the other hand, for the non-activated biotin-arrayed surface, the non-activated biotin should not bind with the amino group. The results obtained when the OIM slide with $10 \mu \mathrm{g} / \mathrm{mL}$ arrays of NHS-activated and non-activated biotin was immersed in $1.0 \mu \mathrm{g} / \mathrm{mL}$ of Cy5-streptavidin are shown in Fig. 2.

As expected, the NHS-activated biotin-arrayed surface exhibited a higher fluorescence intensity than the non-activated biotin-arrayed surface. This higher intensity is attributed to the formation of a biotin-streptavidin conjugate, which confirms that NHS-activated biotin could be immobilized on the OIM slide. The non-activated biotin-arrayed surface exhibited a slightly higher fluorescence intensity than the unarrayed surface because of the nonspecific adsorption of non-activated biotin. The small difference in the fluorescence intensity between these two surfaces was also predictable. From the fluorescence intensity for the three surfaces, we concluded that biotin was immobilized on the OIM slide.
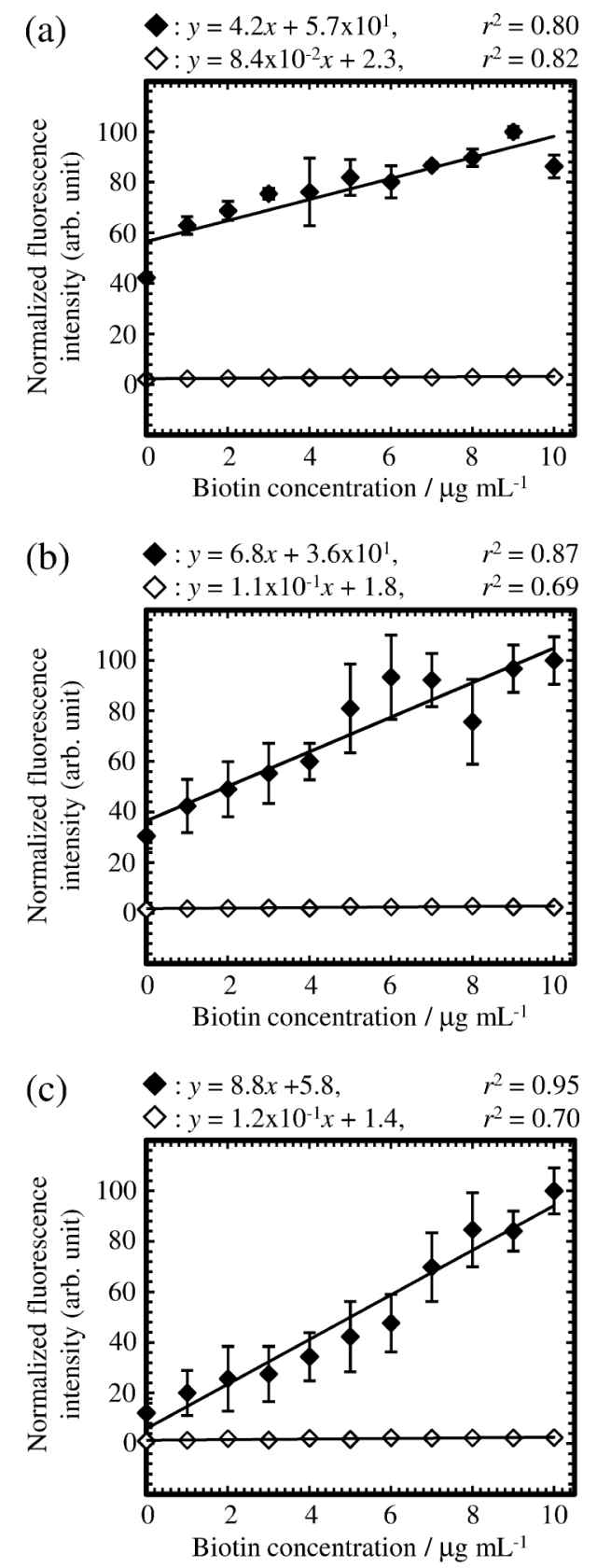

Fig. 3 Solution assays with the OIM and glass slides. (a) Fluorescein-avidin, (b) Cy3-streptavidin, (c) Cy5-streptavidin. The maximum fluorescence intensity with the OIM slide was normalized as 100. The fluorescence intensity was averaged for five spots. The filled and empty squares represent the OIM and glass slides, respectively. The straight lines and the error bars indicate the standard curve and the standard deviation, respectively.

\section{Solution assays of avidin/streptavidin}

Next, we performed solution assays of avidin/streptavidin. The dye-labeled avidin/streptavidin concentrations were $10 \mu \mathrm{g} / \mathrm{mL}$ for fluorescein-avidin and $1.0 \mu \mathrm{g} / \mathrm{mL}$ for $\mathrm{Cy} 3-$ and Cy5-streptavidin. Here, note the fluorescein-avidin concentration. When the fluorescein-avidin was $1.0 \mu \mathrm{g} / \mathrm{mL}$, a distinct fluorescence from the NHS-activated biotin-arrayed surface could not be detected with the glass slide. This is attributed to a high nonspecific adsorption by both the sugar chain structure, ${ }^{33}$ and the high isoelectric point of avidin. ${ }^{34}$ Thus, the fluorescein-avidin concentration was increased to 
Table 1 Fluorescence enhancement factor obtained with different assay type

\begin{tabular}{lcc}
\hline \multirow{2}{*}{ Dye } & \multicolumn{2}{c}{ Fluorescence enhancement factor } \\
\cline { 2 - 3 } & Solution assay & Spotting assay \\
\hline Fluorescein & 50.0 & 51.8 \\
Cy3 & 61.8 & 67.1 \\
Cy5 & 73.3 & 71.4 \\
\hline
\end{tabular}

ten-times greater than Cy3- and Cy5-streptavidin, so that the fluorescence for the glass slide could be detected by increasing the binding amounts of avidin and biotin.

The results of the solution assay are shown in Fig. 3. The OIM slide exhibited a significantly higher fluorescence intensity for all dyes compared with the glass slide. It was found that the fluorescence intensities for the OIM and glass slides are relatively proportional up to $10 \mu \mathrm{g} / \mathrm{mL}$ of NHS-activated biotin concentration. We interpreted this linear increase as the concentration dependence of immobilized biotin.

Large standard errors are attributed to the inhomogeneity among fluorescent spots that occurred during the experimental processes. A higher amount of nonspecific adsorption for the fluorescein-avidin is considered due to the concentration of ten-times greater than those of Cy3- and Cy5-streptavidin.

The enhancement factor of the fluorescence was then calculated from the slope of the standard curve for the fluorescence intensities obtained with the OIM and glass slides. The enhancement factors are summarized in Table 1. It was found that the fluorescence intensity with the OIM slide achieved an enhancement of approximately 50.0-fold for fluorescein, 61.8-fold for $\mathrm{Cy} 3$, and 73.3-fold for Cy5 compared with the glass slide.

These enhancements include the difference in the immobilization amount of NHS-activated biotin onto the OIM and glass slides. As described in the following section, the immobilization amount of NHS-activated biotin onto the OIM slide was approximately 1.9-fold higher than that onto the glass slide. It was found that the fluorescence intensity with the OIM slide reestimated with the consideration for the immobilization amount is enhanced by approximately 26.3 -fold for fluorescein, 32.5-fold for Cy3, and 38.6-fold for Cy5. These results suggest that the OIM slide can be effectively applied in various biomolecular analyses, including the biotin-avidin/streptavidin system.

\section{Estimation of the nonspecific adsorption amount}

Before evaluating the concentration dependence of dye-labeled avidin/streptavidin using the spotting assay, the validity of this assay method was examined. In general, the dryness of a target solution on a substrate surface is known to cause a considerable amount of nonspecific adsorption. Because the spotting assay spots the target solution on the surface, it was predicted that this method would result in significant nonspecific adsorption. To establish that the fluorescence from the dye is not due to nonspecific adsorption, the amount of nonspecific adsorption of the dye-labeled avidin/streptavidin caused by the spotting method was examined.

Each $10 \mu \mathrm{g} / \mathrm{mL}$ of NHS-activated and non-activated biotin was arrayed onto the aminated OIM slide. After the biotin immobilization process was performed, $1.0 \mu \mathrm{g} / \mathrm{mL}$ of Cy5-streptavidin was spotted onto three different surfaces: the NHS-activated biotin-arrayed surface, the non-activated

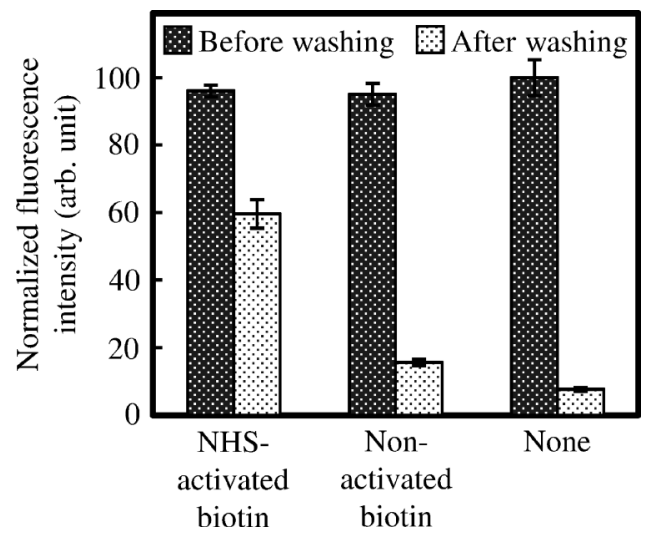

Fig. 4 Nonspecific-adsorption amount of Cy5-streptavidin onto the OIM slide surface before and after washing. The fluorescence intensity with the unarrayed surface before washing was normalized as 100 . The fluorescence intensity was averaged for five spots. Error bars indicate the standard deviation.

biotin-arrayed surface, and the unarrayed surface. The fluorescence from Cy5 was measured using a fluorescence scanner. At this stage, because Cy5-streptavidin remained physically adsorbed on the OIM slide, the OIM slide was immersed in the Tris- $\mathrm{HCl}$ buffer to bind it to the immobilized biotin. After washing, the fluorescence from Cy5 was measured again. The measurement results before and after washing are shown in Fig. 4.

Before washing, the fluorescence intensities obtained for three surfaces were almost the same, because Cy5-streptavidin physically adsorbed on the surface as a result of the drying of the spot solution. On the other hand, the fluorescence intensity after washing changed substantially. At this time, the NHS-activated biotin-arrayed surface had NHS-activated biotin immobilized onto the OIM slide. The non-activated biotin-arrayed surface had a small amount of nonspecifically adsorbed non-activated biotin.

The fluorescence intensity for the non-activated biotin-arrayed surface was approximately $26 \%$ of that for the NHS-activated biotin-arrayed surface. Moreover, the intensity for the unarrayed surface was reduced to approximately $13 \%$, because biotin does not exist on the surface. The nonspecific adsorption rate of Cy5-streptavidin onto the unarrayed surface almost corresponded with the result for the unarrayed surface, as shown in Fig. 2. This correspondence supports the contention that the spotting assay can obtain results equivalent to those of the solution assay. Thus, we determined that the fluorescence from the dye for the spotting assay was not due to the nonspecific adsorption of the target.

Then, the same experiments were performed with the glass slide to estimate the immobilization amount of NHS-activated biotin. The amount of Cy5-streptavidin on the glass slide before washing was the same as that on the OIM slide, while the amount of Cy5-streptavidin after washing varied by the amount of NHS-activated biotin immobilized onto each a slide. From the difference in the fluorescence intensities before and after washing, the immobilization amount of NHS-activated biotin for each slide was estimated.

The fluorescence intensity with the OIM slide after washing was reduced by approximately $62 \%$, compared with that before washing (Fig. 4). On the other hand, the fluorescence intensity with the glass slide was reduced by approximately $32 \%$ (data not shown). From these results, it was found that the 
immobilization amount of NHS-activated biotin onto the OIM slide was approximately 1.9 -fold higher than the glass slide.

\section{Spotting assays of avidin/streptavidin}

Finally, we performed spotting assays of avidin/streptavidin. The dye-labeled avidin/streptavidin concentrations were $0-10 \mu \mathrm{g} / \mathrm{mL}$ for fluorescein-avidin and $0-1.0 \mu \mathrm{g} / \mathrm{mL}$ for Cy3and Cy5-streptavidin. The results of the spotting assays are shown in Fig. 5.

Similar to the solution assay, the fluorescence intensities obtained with the OIM slide for all dyes were significantly higher than those obtained with the glass slide. It was found that the fluorescence intensities for the OIM and glass slides increased linearly up to concentrations of $10 \mu \mathrm{g} / \mathrm{mL}$ for fluorescein-avidin and $1.0 \mu \mathrm{g} / \mathrm{mL}$ for Cy3- and Cy5-streptavidin. We interpreted these monotonous increases as the concentration dependence of dye-labeled avidin/streptavidin. Compared to the results of the solution assays, the range of the error in the fluorescence intensity due to the inhomogeneity among fluorescent spots was very small. The small error range confirmed that the spotting assay can more quantitatively estimate the sensitivity of the avidin/streptavidin detections than a common analytical method.

The fluorescence enhancement factor was then calculated from the slope of the standard curve for the fluorescence intensities obtained with the OIM and glass slides. The enhancement factors are summarized in Table 1. It was found that the fluorescence intensity obtained with the OIM slide was improved by approximately 51.8-fold for fluorescein, 67.1-fold for Cy3, and 71.4-fold for Cy5 compared with the glass slide. Moreover, it was found that the fluorescence intensity with the OIM slide, re-estimated with the consideration for the immobilization amount of NHS-activated biotin, was enhanced by approximately 27.3-fold for fluorescein, 35.3-fold for Cy3, and 37.6-fold for Cy5.

However, we expected that the fluorescence enhancement when considered the immobilization amount will be around 80 -fold. In our previous reports, the fluorescence enhancement of spin-coated rhodamine $\mathrm{B}$ on the OIM slide achieved 200 -fold, ${ }^{22}$ while that of spotted rhodamine B was 80 -fold. ${ }^{22}$ This decrease of the fluorescence enhancement was due to difference of the deposition method of rhodamine B. The results of this study indicate that less than a 40-fold enhancement is probably due to changes of the surface conditions by the immobilized biotin.

The fluorescence enhancement factor for all dyes almost corresponded with the results of the solution assays. The magnitude of these enhancement factors was comparable to, or more than, those obtained by other competing groups. The enhancement factor also increased in the order of fluorescein $<\mathrm{Cy} 3<\mathrm{Cy} 5$. This order agrees well with that we reported previously, ${ }^{23}$ thereby validating the data obtained in this study. Based on fluorescent labeling, we conclude that the OIM slide can be used as a substrate for highly sensitive biomolecular analysis.

\section{Conclusions}

In the present work, we demonstrated highly sensitive fluorescence detection of avidin/streptavidin using an OIM slide fabricated with $\mathrm{Ag}$ and $\mathrm{Al}_{2} \mathrm{O}_{3}$. The detection sensitivities of avidin/streptavidin labeled with fluorescein, Cy3, and Cy5 were estimated by two methods. In the solution assay, the detection sensitivity of the avidin/streptavidin on the OIM slide improved

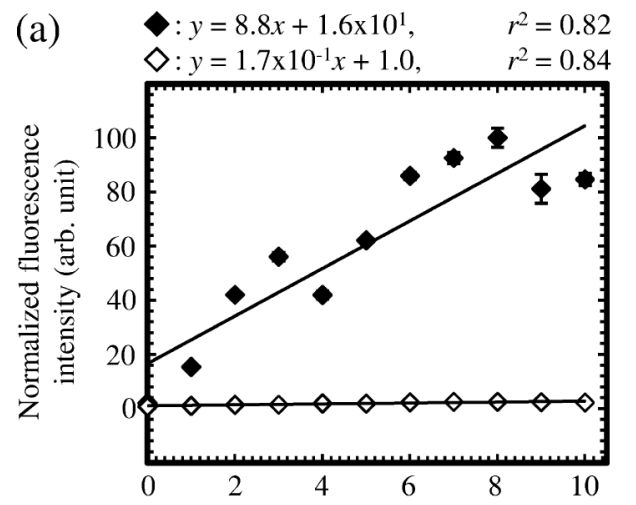

Fluorescein-avidin concentration $/ \mu \mathrm{g} \mathrm{mL}^{-1}$
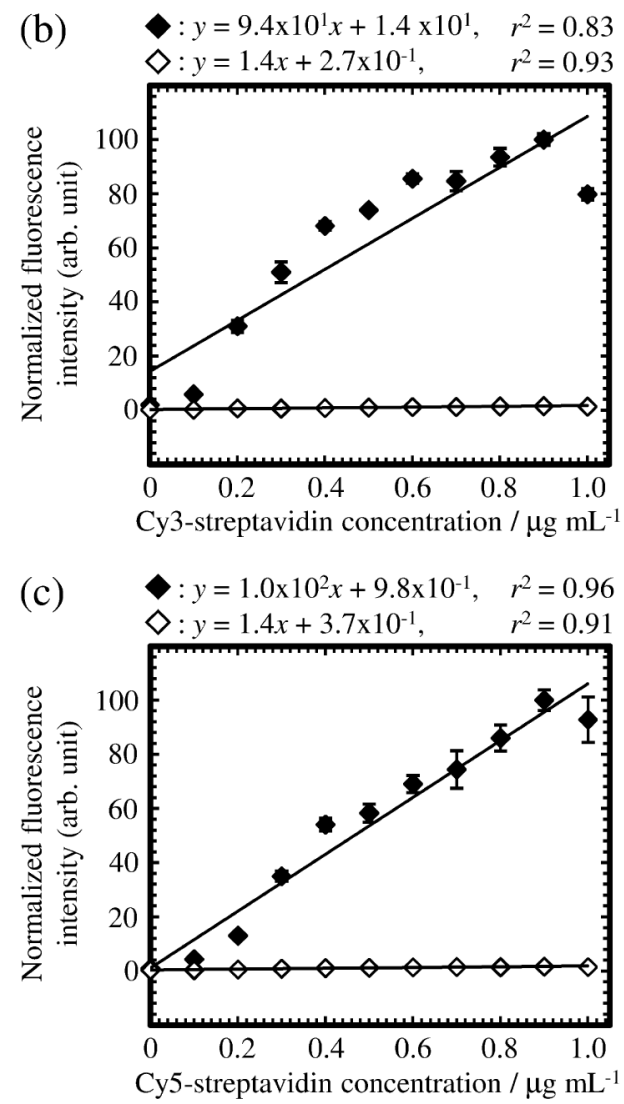

Fig. 5 Spotting assays with the OIM and glass slides. (a) Fluorescein-avidin, (b) Cy3-streptavidin, (c) Cy5-streptavidin. The maximum fluorescence intensity with the OIM slide was normalized as 100. The fluorescence intensity was averaged for five spots. The filled and empty squares represent the OIM and glass slides, respectively. The straight lines and the error bars indicate the standard curve and the standard deviation, respectively.

by two orders of magnitude for all dyes compared with the glass slide. In addition, the detection sensitivity of avidin/streptavidin using the spotting assay achieved almost the same improvement as the solution assay. We also established that compared with common analytical methods, such as the solution assay, the spotting assay can more quantitatively estimate the sensitivity of the avidin/streptavidin detections.

The OIM slide provides a high magnitude of fluorescence enhancement despite its simple structure. This simple structure allows easy fabrication of the OIM slide, which leads to high reproducibility and low cost. These features indicate that the 
OIM slide would be a promising alternative to traditional substrates for biomolecular analysis. Using the OIM slide would contribute to further progress in biomedical research and medical diagnostics.

\section{Acknowledgements}

This work was supported by a Grant-in-Aid for Scientific Research (C) from the Japan Society for the Promotion of Science.

\section{References}

1. S. M. Yoo, J. H. Choi, S. Y. Lee, and N. C. Yoo, J. Microbiol. Biotechnol., 2009, 19, 635.

2. D. A. Hall, J. Ptacek, and M. Snyder, Mech. Ageing Dev., 2007, 128, 161.

3. S. Y. Kim, J. Yu, S. J. Son, and J. Min, Ultramicroscopy, 2010, 110, 659.

4. S. O. Meade, M. Y. Chen, M. J. Sailor, and G. M. Miskelly, Anal. Chem., 2009, 81, 2618.

5. T. Shtoyko, E. G. Matveeva, I-F. Chang, Z. Gryczynski, E. Goldys, and I. Gryczynski, Anal. Chem., 2008, 80, 1962.

6. C. Oillic, P. Mur, E. Blanquet, G. Delapierre, F. Vinet, and T. Billon, Mater. Sci. Eng., C, 2007, 27, 1500.

7. A. Dorfman, N. Kumar, and J.-I. Hahm, Langmuir, 2006, $22,4890$.

8. K. Aslan, J. R. Lakowicz, and C. D. Geddes, J. Phys. Chem. $B, \mathbf{2 0 0 5}, 109,6247$.

9. E. G. Matveeva, I. Gryczynski, A. Barnett, Z. Leonenko, J. R. Lakowicz, and Z. Gryczynski, Anal. Biochem., 2007, $363,239$.

10. C. R. Sabanayagam and J. R. Lakowicz, Nucleic Acids Res., 2007, 35, e13.

11. J. R. Lakowicz, C. D. Geddes, I. Gryczynski, J. Malicka, Z. Gryczynski, K. Aslan, J. Lukomska, E. Matveeva, J. Zhang, R. Badugu, and J. Huang, J. Fluoresc., 2004, 14, 425.

12. P. Takmakov, I. Vlassiouk, and S. Smirnov, Anal. Bioanal. Chem., 2006, 385, 954.

13. A. Ressine, D. Finnskog, J. Malm, C. Becker, H. Lilja, G. M. Varga, and T. Laurell, NanoBiotechnology, 2005, 1, 93.

14. F. Bessueille, V. Dugas, V. Vikulov, J. P. Cloarec, E. Souteyrand, and J. R. Martin, Biosens. Bioelectron., 2005, $21,908$.
15. K. Kuwabara, M. Ogino, T. Ando, and A. Miyauchi, Appl. Phys. Lett., 2008, 93, 033904.

16. B. R. Murthy, J. K. K. Ng, E. S. Selamat, N. Balasubramanian, and W. T. Liu, Biosens. Bioelectron., 2008, 24, 723.

17. J. Shi, C. Peroz, D. Peyrade, J. Salari, M. Belotti, W. H. Huang, and Y. Chen, Microelectron. Eng., 2006, 83, 1664.

18. W. R. Holland and D. G. Hall, Opt. Lett., 1985, 10, 414.

19. K. G. Sullivan, O. King, C. Sigg, and D. G. Hall, Appl. Opt., 1994, 33, 2447.

20. K. G. Sullivan and D. G. Hall, J. Opt. Soc. Am. B, 1997, 14, 1149.

21. K. G. Sullivan and D. G. Hall, J. Opt. Soc. Am. B, 1997, 14, 1160.

22. T. Akimoto, M. Yasuda, and I. Karube, Appl. Opt., 2008, 47, 3789.

23. T. Akimoto and M. Yasuda, Appl. Opt., 2010, 49, 80.

24. E. L. Moal, E. Fort, S. Lévêsque-Fort, F. P. Cordelières, M.-P. Fontaine-Aupart, and C. Ricolleau, Biophys. J., 2007, 92, 2150.

25. E. L. Moal, E. Fort, S. Lévêque-Fort, A. Janin, H. Murata, F. P. Cordelières, M.-P. Fontaine-Aupart, and C. Ricolleau, J. Biomed. Opt., 2007, 12, 024030.

26. V. Marino, C. Galati, and C. Arnone, J. Biomed. Opt., 2008, 13, 054060 .

27. C. Oillic, P. Mur, E. Blanquet, G. Delapierre, F. Vinet, and T. Billon, Biosens. Bioelectron., 2007, 22, 2086.

28. M. Bras, V. Dugas, F. Bessueille, J. P. Cloarec, J. R. Martin, M. Cabrera, J. P. Chauvet, E. Souteyrand, and M. Garrigues, Biosens. Bioelectron., 2004, 20, 797.

29. R. J. Redkar, N. A. Schultz, V. Scheumann, L. A. Burzio, D. E. Haines, E. Metwalli, O. Becker, and S. D. Conzone, J. Biomol. Tech., 2006, 17, 122.

30. H. Choumane, N. Ha, C. Nelep, A. Chardon, G. O. Reymond, C. Goutel, G. Cerovic, F. Vallet, and C. Weisbuch, Appl. Phys. Lett., 2005, 87, 031102.

31. B. Fouqué, B. Schaack, P. Obeïd, S. Combe, S. Gétin, P. Barritault, P. Chaton, and F. Chatelain, Biosens. Bioelectron., 2005, 20, 2335.

32. T. S. Eriksson, A. Hjortsberg, G. A. Niklasson, and C. G. Granqvist, Appl. Opt., 1981, 20, 2742.

33. Y. Hiller, J. M. Gershoni, E. A. Bayer, and M. Wilchek, Biochem. J., 1987, 248, 167.

34. H. Kobayashi, H. Sakahara, K. Endo, M. Hosono, Z.-S. Yao, S. Toyama, and J. Konishi, Jpn. J. Cancer Res., 1995, 86, 310. 